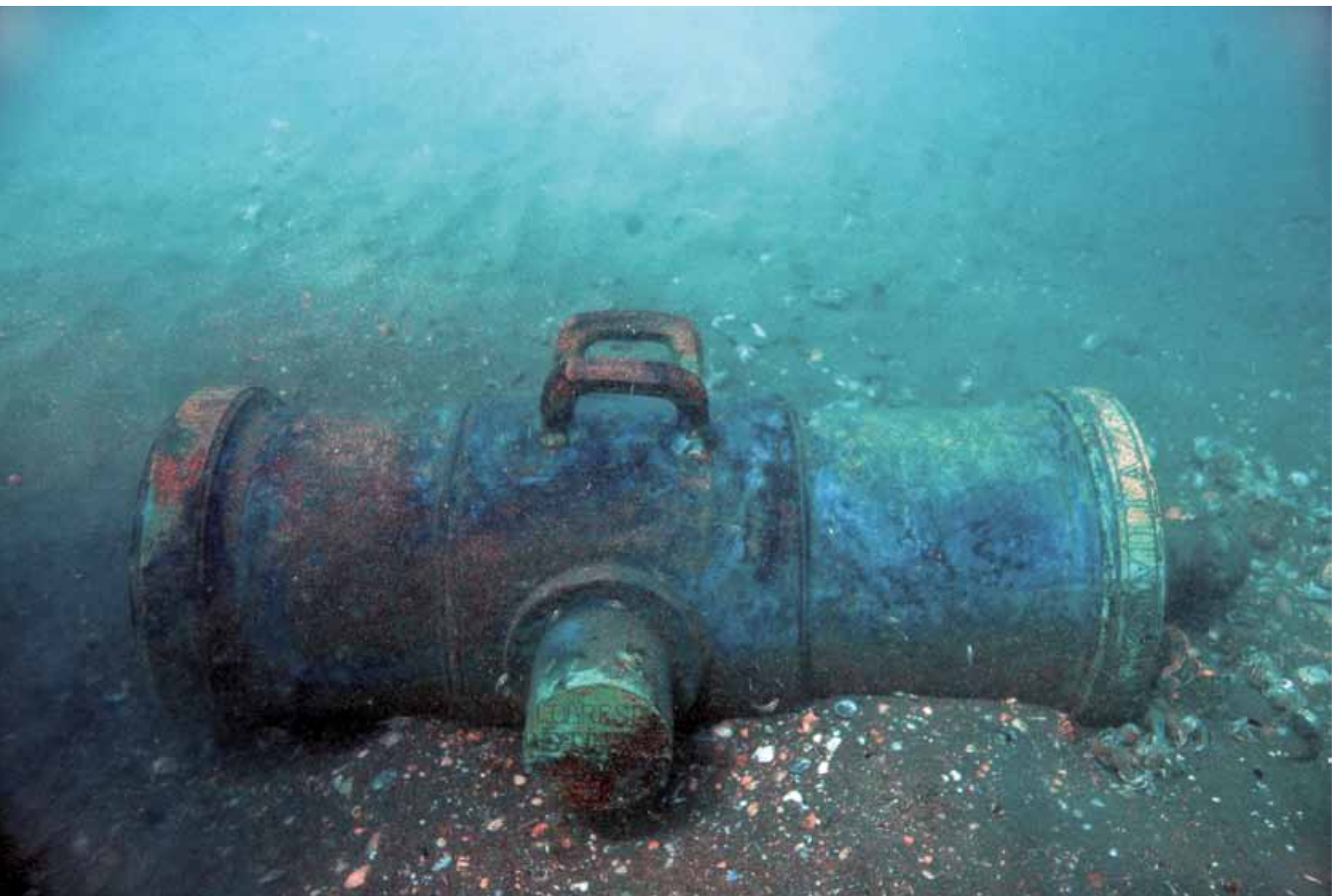

Cañón. Fuente: CAS-IAPH 


\title{
Estabilización de objetos metálicos de procedencia subacuática por métodos electroquímicos
}

Abel Bocalandro Rodríguez,

Licenciado en Química

\begin{abstract}
Resumen
Los tratamientos de estabilización electrolítica están basados en la extracción de los iones cloruros, principales causantes del deterioro de los objetos metálicos en ambientes marinos, mediante la aplicación de una diferencia de potencial con el que se consigue aumentar la difusión de dichos iones mediante la reducción de los productos de corrosión formados en el objeto. Además, durante el tratamiento electroquímico, en el objeto metálico va a variar su potencial de oxidación favoreciendo su pasivación frente a los procesos de corrosión que sufren tanto en el yacimiento como en la post-excavación.
\end{abstract}

\section{Palabras clave}

Arqueología subacuática / Centro de Arqueología Subacuática / Conservación / Corrosión / Reducción electrolítica / Eliminación de cloruros / Instituto Andaluz del Patrimonio Histórico / Investigación / Metal / Metodologia / Tratamiento electroquimico 


\section{INTRODUCCIÓN}

La mayoría de los metales en ambientes marinos son susceptibles de ser deteriorados por procesos corrosivos activados principalmente por el agua, las sales y los gases en disolución, en especial el oxígeno. Estos procesos corrosivos que ocurren en el agua de mar son de naturaleza electroquímica y dan como resultado la formación sobre la superficie metálica de una multitud de zonas anódicas y zonas catódicas. La creación de estas celdas electroquimicas se ve favorecida por la alta conductividad iónica que presenta el medio marino, el cual actúa de electrolito. En estas celdas electroquímicas los electrones que ceden los metales en las zonas anódicas son consumidos en las zonas catódicas de la superficie metálica por la reducción del oxígeno presente en el medio. Este oxígeno que está presente en el agua de mar, aún a grandes profundidades, es la fuente principal de la reacción catódica que promueve la reacción de corrosión por disolución del metal en el ánodo. La reducción de oxígeno produce iones hidróxidos $\left(\mathrm{OH}^{-}\right)$que aumentan el pH en el cátodo; en ausencia de oxígeno, la otra reacción catódica posible es la reducción del catión hidrógeno $\left(\mathrm{H}^{+}\right)$. Las reacciones del metal con el medio van a formar los denominados productos de corrosión, los cuales precipitan sobre la superficie metálica. Estos productos de corrosión, junto a los precipitados insolubles de carbonato de calcio que se crean por un desequilibrio entre las concentraciones de carbonato de calcio y dióxido de carbono que se encuentran disueltos en el agua debido a los cambios de $\mathrm{pH}$, interactúan con el sedimento y los organismos marinos presentes en la superficie de la pieza para formar una densa concreción alrededor del metal.

Tanto el oxígeno como la humedad, una vez que el objeto metálico es extraído, pueden ser controlados mediante distintas técnicas de conservación como, por ejemplo, la aplicación de distintos productos químicos que actúan de capas de protección frente a estos dos factores propulsores de la corrosión metálica. Esto nos hace pensar que el principal agente corrosivo de los objetos metálicos son las sales presentes en el agua de mar, fundamentalmente los iones cloruros que penetran en el

\section{Mediante la optimización de las técnicas electrolíticas se ha conseguido una mejora de la estabilización del objeto}

interior de la pieza, atraídos por las zonas anódicas, llevando al objeto a un estado de inestabilidad, el cual puede conducir a la desaparición total de la pieza metálica. Por ello, los complejos inorgánicos con presencia de aniones cloro en cualquiera de sus formas deben ser removidos de la microestructura interna del objeto metálico.

En el caso de las piezas de hierro recuperadas del medio marino, los mayores daños sobre los objetos están causados por los cloruros de hierro. Tras la exposición a la humedad y al oxígeno, estos cloruros se hidrolizan para formar óxido de hierro o hidróxido de hierro y ácido clorhídrico. El ácido clorhídrico, a su vez, oxida el resto de metales no corroídos a cloruros ferrosos e hidrógeno, o a cloruro férrico y agua. Se desencadenan una serie de reacciones que pueden prolongarse hasta la desaparición completa del metal. Cuando los óxidos de hierro son transformados en hidróxidos (FeOOH), resulta que coexisten varias especies de hidróxidos como son: goetita ( $\alpha-\mathrm{Fe} \mathrm{OOH})$, bastante estable y de color marrón; lepidocrocita, algo menos estable que la goetita y de coloración marrón-rojiza; y la akaganeita ( $\beta$ - $\mathrm{FeOOH})$. Este último oxihidróxido que presenta una coloración marrónamarillenta es el principal desestabilizador y responsable último de la destrucción de los objetos de hierro tras ser exhumados, ya que posee la capacidad de albergar en su estructura cristalina a los cloruros tras su combinación. Está compuesto principalmente por cloruro férrico, que queda retenido en su estructura en grandes cantidades. La akaganeita es un mineral que posee una estructura túnel del tipo del mineral holandita. Posee una gran capacidad de intercambio de iones de su estructura tipo túnel con los iones $\mathrm{Cl}^{-}$y $\mathrm{OH}^{-}$. Al igual que ocurre con los otros alfa y gamma oxihidróxidos, no figura ningún átomo de cloro en su fórmula química pero, como resultado de intercambios iónicos, sí pueden aparecer intercalados entre su estructura cristalina. Hasta tal punto que estos productos guardan en su interior una buena parte de los cloruros que contiene un objeto de hierro.

Los aniones cloruros no solamente actúan en la corrosión del metal provocando alteraciones químicas, las cuales se pueden detectar visualmente por los productos de corrosión formados, sino que también pueden ejercer una alteración de tipo física sobre el metal por eflorescencia salina. Los iones cloruros inicialmente disueltos en la humedad propia del suelo o mar pueden reaccionar con un material poroso, como es el hierro, y actuar en su proceso de corrosión. Y después, por descenso del indice de humedad, pueden cristalizar en los poros o entre las grietas del hierro. Esta cristalización conlleva un aumento de volumen producido durante el crecimiento del cristal, creando tensiones internas que provocan nuevos agrietamientos o desprendimientos de capas superficiales de productos de corrosión.

Ya que la corrosión de los metales en ambientes húmedos es de naturaleza electroquímica, una aproximación lógica para intentar parar la corrosión sería mediante los métodos electroquímicos. Los métodos electroquímicos para la protección contra la corro- 
sión requieren de un cambio en el potencial del metal para prevenir o al menos disminuir su disolución.

La técnica de estabilización electrolítica utilizada en el tratamiento de los materiales de origen metálico de procedencia subacuática se fundamenta en los principios de la denominada protección catódica con ánodo de sacrificio.

La protección catódica consiste en polarizar catódicamente el metal. En este caso el metal a proteger es convertido en el cátodo de una celda electroquímica. Podemos decir que la protección catódica es un tipo de protección contra la corrosión, en la cual el potencial del electrodo del metal en cuestión se desplaza en la dirección negativa. Partiendo de zonas donde la corrosión está favorecida, se intenta polarizar al metal para llevarlo, si es posible, a una zona de inmunidad, es decir, donde termodinámicamente no se da la corrosión. Ahora bien, modificar el potencial mediante una polarización catódica hasta un punto de inmunidad es un gasto en corriente muy grande, por lo que, normalmente, lo que se pretende es llegar a zonas donde la velocidad de corrosión sea menor.

De acuerdo con el diagrama de Pourbaix, donde se representa potencial frente a $\mathrm{pH}$, hay un camino para prevenir la corrosión mediante un cambio de potencial. En estos diagramas se tienen todos los datos de la termodinámica de la oxidación de los metales. Son característicos de cada metal y, en función del medio y de las condiciones ambientales, estos diagramas varían.

Aplicando una corriente catódica, el potencial del espécimen a proteger se desplazará en la dirección negativa hacia la zona de inmunidad, en la cual el metal termodinámicamente se encuentra estable (no sufre en principio procesos de corrosión). Para lograr este desplazamiento del potencial en la dirección negativa, el objeto que se corroe se une eléctricamente a un metal más negativo en la serie electroquímica, un ánodo, formando una celda galvánica en la que actuará como cátodo.

Con la protección catódica se consigue eliminar todos los ánodos de la superficie metálica, es decir, se elimina todas las zonas donde se están llevando a cabo procesos de oxidación en la superficie del objeto metálico, haciendo que la superficie metálica actúe como cátodo.

Para volver catódica a la superficie metálica el procedimiento seguido ha sido la utilización de ánodos de sacrificios. Esto consiste, básicamente, en conectar la estructura a proteger a un metal más activo que el que estamos protegiendo. De esta manera obtendremos un par galvánico. Como en todo par galvánico, aumentará la velocidad de oxidación del metal más activo, mientras que el más noble, que es el que vamos a proteger, disminuye su velocidad de corrosión. De esta manera tendremos que el metal que actúa como ánodo se consume, por lo que hay que ir chequeando su potencial para ir controlando que no se agote, ya que si no se desharía el par galvánico y el metal arqueológico se corroería a su velocidad normal.

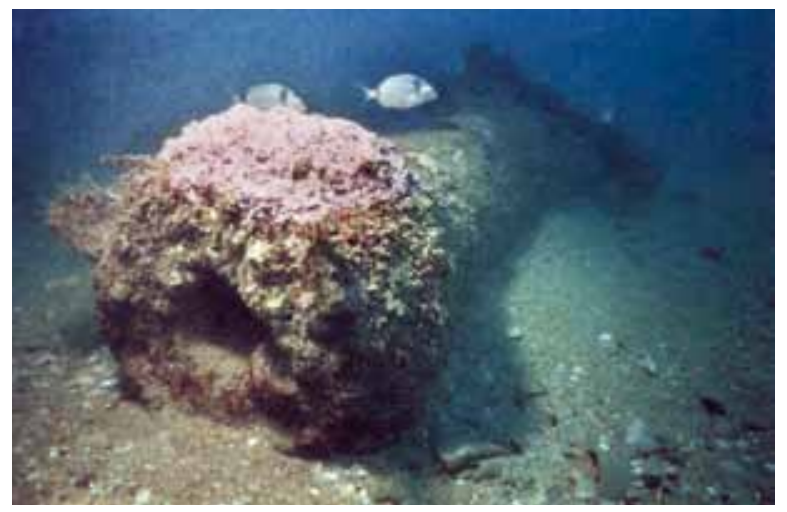

Cañones de hierro concrecionados. Fuente: CAS-IAPH

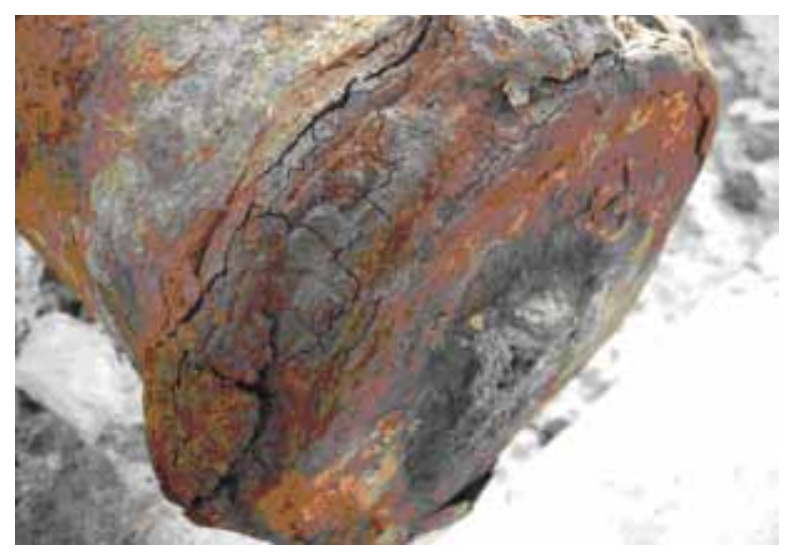

Detalle de la boca de un cañón con varias grietas y fragmentos a medio desprenderse. Fuente: CAS-IAPH

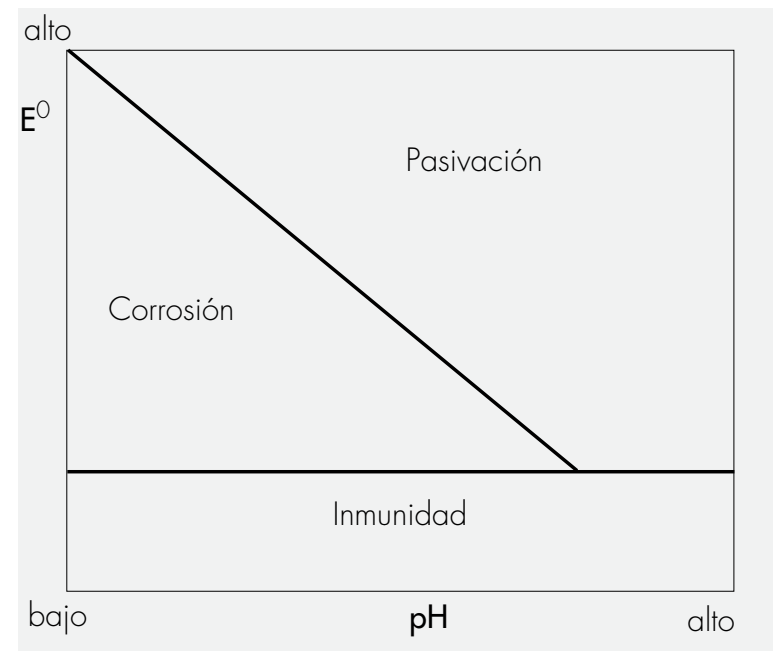

Diagrama de Pourbaix. Fuente: CAS-IAPH

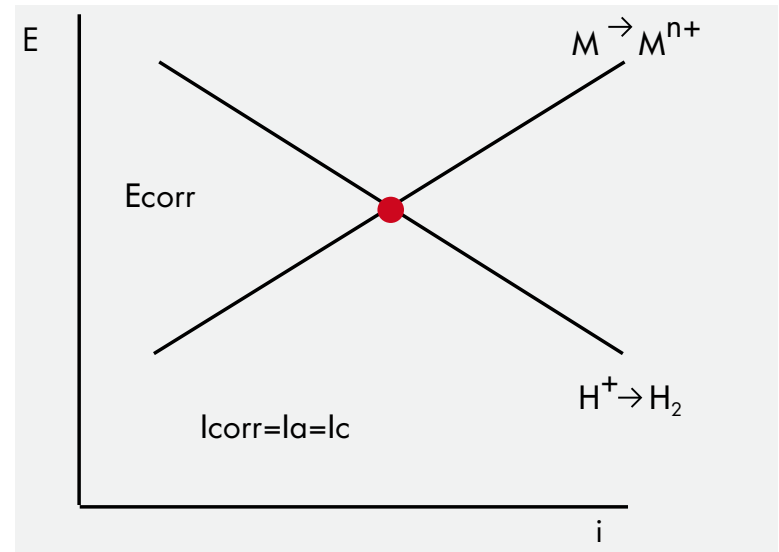

Diagrama de Evans. Fuente: CAS-IAPH 
Este sistema, que se conoce como protección catódica con ánodos de sacrificio, consiste realmente en la creación de una celda electrolítica en la que la pieza metálica a proteger actúe forzosamente de cátodo, mientras que el metal anódico se sacrifica.

Desde un punto de vista cinético, en un sistema cualquiera en el cual tenga lugar el fenómeno de corrosión, existe un balance perfecto entre las reacciones anódicas y catódicas sobre la superficie del metal. Esto se puede observar gráficamente mediante los diagramas de Evans, en donde se relaciona la densidad de corriente con el potencial.

Para un metal $M$, en el diagrama de Evans, las reacciones que tienen lugar en las zonas anódicas y catódicas son las siguientes:

ánodo: $\mathrm{M} \rightarrow \mathrm{M}^{\mathrm{n}+}+\mathrm{ne}^{-}$

cátodo: $2 \mathrm{H}^{+}+2 \mathrm{e}^{-} \rightarrow \mathrm{H}_{2}$

Polarizando católicamente lo que estamos haciendo es que la disminuya mientras que $/ c$ aumente.

El objetivo del tratamiento electroquímico es la estabilización de los objetos por la eliminación de cloruros de los productos de corrosión mediante su reducción.

Las diferentes técnicas de limpiezas de cloruros han mostrado que el paso limitante en la liberación de estos iones es su difusión desde los productos de corrosión. Lo que se persigue con la aplicación de las técnicas de estabilización electrolítica es la manera de aumentar la velocidad de difusión de los cloruros presentes en los productos de corrosión, y esto se consigue transformando los complejos inorgánicos productos de la corrosión en otros más densos mediante su reducción.

North y Pearson, en 1978, hicieron un estudio acerca de la velocidad de difusión de los iones cloruros desde el interior del objeto hacia la solución, y de los parámetros que podian influir en la movilidad iónica de estos aniones.

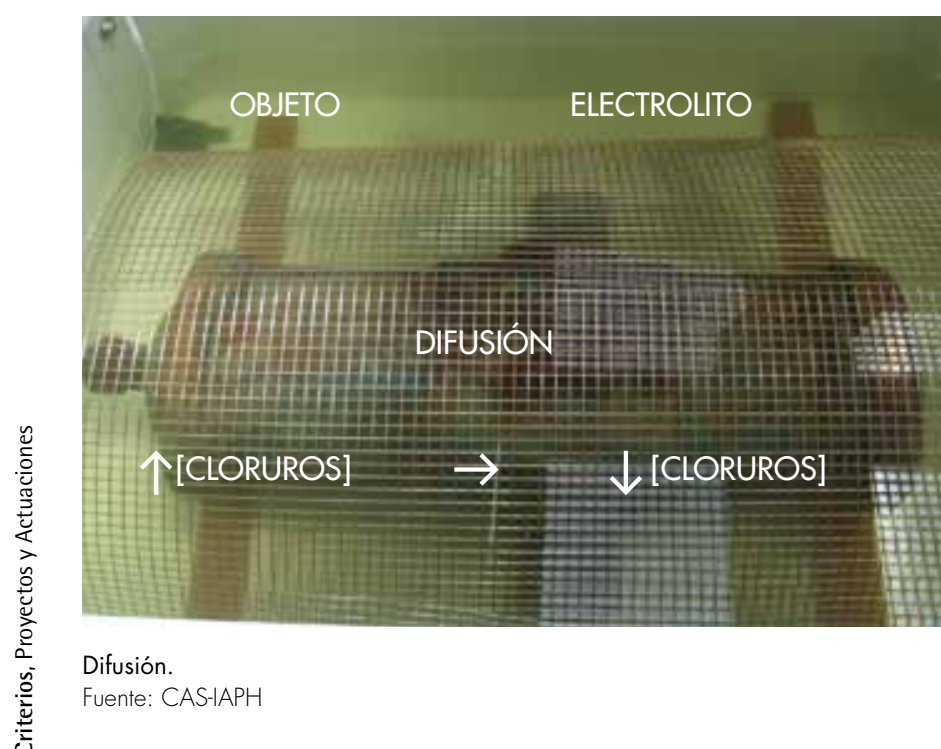

Cuando en un sistema termodinámico hay un gradiente de concentraciones, se origina un flujo irreversible de materia, desde las altas concentraciones a las bajas. A este flujo se le llama difusión. La difusión tiende a devolver al sistema a su estado de equilibrio, de concentración constante. La ley de Fick nos dice que el flujo difusivo que atraviesa una superficie ( $\mathrm{J}$ en $\mathrm{mol} \mathrm{cm}^{-2} \mathrm{~s}^{-1}$ ) es directamente proporcional al gradiente de concentración, es decir, la velocidad de difusión es directamente proporcional a la diferencia de concentraciones. Esto viene representado mediante la siguiente ecuación:

$J=-D(d c / d x)$

Donde J es igual al flujo o corriente neta de átomos; D es el coeficiente de difusión (en $\mathrm{cm}^{-2} \mathrm{~s}^{-1}$ ); y dc/dx es el gradiente de concentración. Se utiliza un signo negativo porque la difusión tiene lugar desde una concentración mayor a otra menor, es decir, existe un gradiente negativo. Esta ecuación se llama primera ley de difusión de Fick, y corresponde a aquellas situaciones en que no hay cambios en el transcurso del tiempo.

El coeficiente de difusión depende de:

- tipo de mecanismo de difusión

- temperatura

- estructura cristalina

- tipos de imperfecciones en la red cristalina

- concentración de la especie que difunde

Los dos mecanismos importantes mediante los cuales ocurren estos procesos de difusión son la difusión por vacancia y la difusión intersticial:

- Difusión por vacancia: tanto en la autodifusión como en la difusión de átomos sustitucionales, un átomo puede abandonar su sitio en la red para llenar un puesto vacante cercano, pero en el mismo momento se crea una vacancia en el sitio antes ocupado por él. Al progresar la difusión se observa un flujo de átomos y de vacancias en sentidos opuestos, conocido como difusión por vacancia.

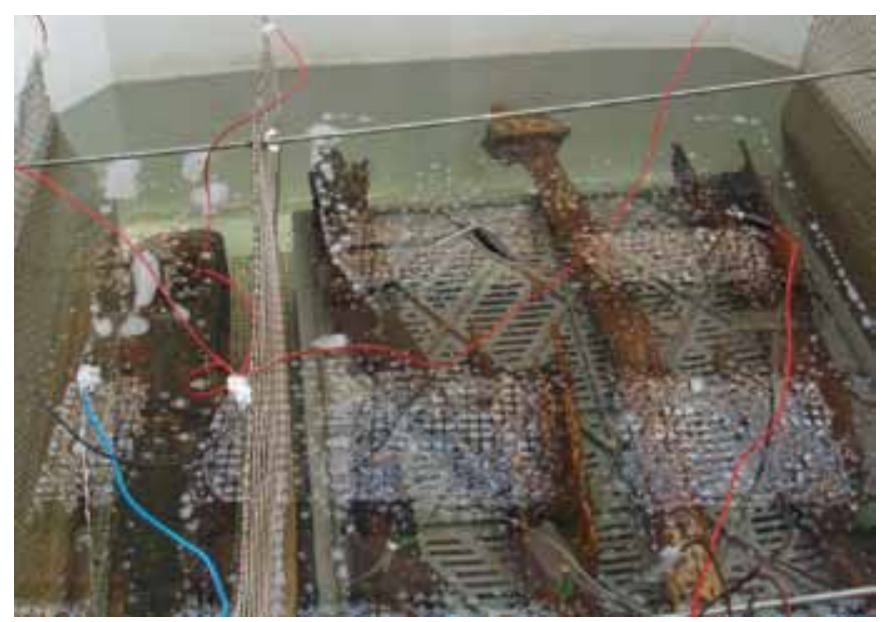

Evolución de hidrógeno por aplicación de altas intensidades. Fuente: CAS-IAPH 
- Difusión intersticial: si en la estructura cristalina está presente un átomo o ión pequeño en un sitio intersticial, éste pasará de un sitio intersticial a otro sin necesidad de que existan vacancias. Este mecanismo de difusión es más rápido que el anterior, ya que el número de sitios intersticiales es mayor que el de vacancias.

La velocidad de difusión aumentará si en el sistema se da un incremento de la temperatura. La dependencia de la velocidad de difusión con la temperatura en muchos casos puede expresarse en términos del coeficiente de Difusividad. Por experimentación se ha encontrado que esta relación de la temperatura con la velocidad de la difusión en muchos sistemas puede ser expresada por el siguiente tipo de ecuación de Arrhenius:

$D=D_{0} \quad e-0 / R T \quad(1)$

Con 0 como la energía de activación, $\mathrm{D}_{0}$ constante para cada sistema de difusión dado (los valores típicos se encuentran tabulados), $R$ constante de los gases $=8,314 \mathrm{~J} /(\mathrm{mol} \mathrm{K})$ y $T=$ Temperatura, $\mathrm{K}$.

Experimentalmente se puede determinar la Energía de activación (0) y la constante de proporcionalidad ( $\left.D_{0}\right)$, calculando el coeficiente de Difusividad a dos temperaturas y expresando la ecuación (1) de Arrhenius en términos de sus logaritmos:

$\operatorname{Ln} D=\operatorname{Ln} D_{0}-0 / R T \rightarrow Y=m X+b$

Los factores que disminuyan la energía de activación incrementarán la difusión porque se requerirá menos energía térmica para vencer la barrera energética. Por lo tanto, la difusión intersticial, con una energía de activación baja, ocurre mucho más rápido que la difusión por vacancias.

En general, la energía de activación será menor para átomos que difunden a través de estructuras cristalinas abiertas que compactas, por lo que la mayoria de estructuras abiertas permiten una difusión más rápida de los átomos. Además, como la energía de activación depende de la fuerza del enlace atómico, será mayor para la difusión de átomos en materiales con alto punto de fusión.

En el caso de la estructura cristalina, la difusividad depende el factor de empaquetamiento, por lo que, en estructuras de tipo BCC que posee un factor de empaquetamiento de 0,68, la difusividad será mayor que una red FCC que posee un factor de empaquetamiento de 0,74. De esta manera, por ejemplo, los átomos de carbono se pueden difundir más fácilmente en una red de hierro BCC que una red FCC.

La porosidad también juega un papel importante en la difusión de los iones. Al incrementar el número de poros en la pieza, aumentará la difusión de los iones.

La Segunda Ley de Fick, derivada de la anterior, $d c / d t=D\left(d^{2} c / d x^{2}\right)$, conocida también como Ecuación de Difusión, es una ecuación diferencial cuya integración nos suministra la concentración de la sustancia que se difunde en función del espacio y el tiempo. Esta ley establece que la velocidad de cambio de la composición de la muestra es igual a la difusividad por la velocidad de cambio del gradiente de concentración.

Otro factor que influye en la difusión son las corrientes de convección. La convección forzada, como es la agitación, tiende a disminuir el grosor de la capa de difusión en la superficie $y$, por tanto, disminuye la polarización por concentración. La convención natural, debida a la diferencia de temperaturas o de densidad, también contribuye al transporte de iones.

La utilización de hidróxido de sodio como electrolito de la celda electrolítica para el tratamiento de piezas metálicas de hierro también afecta a la difusión de los iones cloruros, debido a la alta movilidad de los iones hidróxidos que penetran rápidamente en los productos de corrosión, reaccionan con aquellos compuestos que contienen cloruros y facilitan la liberación de estos iones cloruros que difunden a través de los poros hacia la zona de menor concentración.

La velocidad de difusión también puede variar en función del disolvente utilizado, es decir, cada disolvente tiene unas propiedades físico-química intrínsecas que afectan en mayor o en menor medida a la difusión de los iones. Debido a estas propiedades el agua es el disolvente más utilizado.

\section{MÉTODO Y RESULTADOS}

Los métodos electroquímicos se han aplicado a intensidades bajas de corriente, ya que previenen los procesos de evolución de hidrógeno llevados a cabo en la superficie del metal (sobre el cátodo), con lo que evitamos, de esta manera, el deterioro de la zona grafitizada y, además, conseguimos aumentar la velocidad de extracción de los cloruros al incrementar el área disponible para su difusión.

Si aplicamos altas densidades de corriente, lo que ocurre es que sobre la superficie del cátodo se lleva a cabo una alta evolución de hidrógeno, que, por un lado, actúa de barrera física frenando la difusión de los iones cloruros desde el interior de la pieza metálica hasta el baño electrolitico y, por otro lado, puede fracturar al objeto y dañar la zona grafitizada.

Con el empleo de intensidades bajas de corriente vamos a conseguir que se consolide la zona grafitizada del objeto debido a la reducción de los oxhidróxidos de hierro que la componen, principalmente la akaganeita, que se transforma inicialmente en goetita aFeO $(\mathrm{OH})$ y posteriormente en magnetita $\mathrm{Fe}_{3} \mathrm{O}_{4}$. Este compuesto formado finalmente es más denso que los productos de corrosión iniciales, por lo que se favorece la difusión de los iones cloruros desde el interior de la pieza y se consigue consolidar la 
parte metálica externa evitando posteriores oxidaciones. A su vez, este efecto provoca que la difusión de burbujas de hidrógeno en el interior del metal reduzca volumétricamente esta masa y la haga muy porosa, por lo que tenemos que, por un lado, produce un beneficio, al abrir nuevos accesos al electrolito a través de grietas y poros, pudiendo disolver mayor número de iones cloruros, y, por otra parte, libertad también para el mejor acceso del oxígeno, con el riesgo de favorecer nuevos procesos de corrosión.

La electrolisis a baja polarización, aplicada a piezas de cobre utilizando como electrolito soluciones de sesquicarbonato sódico, permite la extracción de cloruros y reducir a productos de corrosión más estables como son la cuprita y los hidroxicarbonatos.

Las soluciones de sesquicarbonato de sodio son ligeramente alcalinas y neutralizan el ácido clorhídrico liberado por el ataque de los cloruros de cobre. Además, el sesquicarbonato de sodio, debido a su grupo bicarbonato, reacciona con el cobre y produce carbonato básico de cobre, que precipita en la superficie del metal y puede ocupar los poros de la pátina, dos efectos protectores. Para la eliminación de las sales de cloruro de un objeto de bronce, es recomendable el reemplazo frecuente de la solución al 5\% en peso de sesquicarbonato de sodio.

$2 \mathrm{CuCl}$ (nantokita) $+\mathrm{H}_{2} \mathrm{O} \rightarrow \mathrm{Cu}_{2} \mathrm{O}$ (cuprita) $+2 \mathrm{Cl}^{-}+2 \mathrm{H}^{+}$

$2 \mathrm{CuCl}$ (nantokita) $+2 \mathrm{H}_{2} \mathrm{O}+\mathrm{O}_{2} \rightarrow \mathrm{Cu}_{2}(\mathrm{OH})_{3} \mathrm{Cl}$ (paratacamita) $+\mathrm{HCl}$

$4 \mathrm{CuCl}+\mathrm{O}_{2}+8 \mathrm{HCO}_{3}^{-} \rightarrow 4 \mathrm{Cu}\left(\mathrm{CO}_{3}\right)_{2}{ }^{2-}+4 \mathrm{H}^{+}+4 \mathrm{Cl}^{-}+2 \mathrm{H}_{2} \mathrm{O}$

$\mathrm{Cu}_{2}(\mathrm{OH})_{3} \mathrm{Cl}$ (paratacamita) $+4 \mathrm{CO}_{3}{ }^{2-} \rightarrow 2 \mathrm{Cu}\left(\mathrm{CO}_{3}\right)_{2}{ }^{2-}+3 \mathrm{OH}^{-}+\mathrm{Cl}^{-}$

El ión $\mathrm{Cu}\left(\mathrm{CO}_{3}\right)_{2}{ }^{2-}$ se supone que es estable en presencia de iones bicarbonato. Este compuesto precipita. De esta manera, se forma la capa de malaquita $\left[\mathrm{CuCO}_{3} \cdot \mathrm{Cu}(\mathrm{OH})_{2}\right]$.

Para llevar a cabo la aplicación de la técnica de estabilización electrolítica, habrá que formar una celda electrolítica, que estará formada por un cátodo, un ánodo, un electrolito, un baño y una fuente de alimentación.

\section{Cátodo}

El cátodo de la celda electrolítica será la propia pieza que queremos tratar. Para que se lleve a cabo la reacción de reducción en la pieza es necesario que la corriente externa de aplicación llegue hasta el núcleo metálico de la pieza arqueológica. Esto es debido a que los metales son conductores y los productos de corrosión no. Por ello, tendremos que conectarle unos electrodos a la pieza, que serán los que sirvan de intermediarios en la conexión fuente externa-cátodo. La instalación de los electrodos conlleva realizar una incisión más o menos profunda en la pieza para que entren en contacto con el núcleo metálico. Esto supone un pequeño deterioro superficial a la pieza, por lo que se recomienda hacerle la conexión en partes poco vistosas del material, con vistas a una futura exposición del objeto arqueológico.
Para simplificar este daño, más puramente estético que físicoquímico, se ha ido modificando el diámetro de los electrodos y la forma de éstos.

Se han utilizado en principio varillas de 7 y $5 \mathrm{~mm}$ de diámetro, luego han sido sustituidas por tornillos de distintos diámetros (2, 3, $4 \mathrm{~mm}$ ) conectados mediante alambres de acero inoxidable a las conexiones de la fuente externa. También se han tomado como electrodos alambres de acero inoxidable de diámetros muy pequeños directamente en contacto con el núcleo metálico, o sujetos con resina epoxi. Esta resina epoxi se ha utilizado porque, además de servir de sujeción al electrodo, es un material conductor.

El cátodo se conecta con la salida negativa de la fuente de alimentación utilizada.

\section{Ánodo}

Como ánodo se ha empleado una malla de acero inoxidable AISI 316 ( Fe/Cr18/Ni10/Mo 3) para la protección catódica. Los aceros inoxidables son aleaciones de hierro con un mínimo de un 10,5\% de cromo. Se añade molibdeno para aumentar la resistencia a la corrosión especialmente en entornos que contienen cloruros.

El ánodo se coloca alrededor del cátodo y lo más cerca posible.

La malla que actuará como ánodo de sacrificio se conectará con la salida positiva de la fuente de alimentación, con el punto de contacto situado fuera del electrolito para prevenir problemas de corrosión galvánica (las tuercas y los pernos serán de acero inoxidable pero el cable que se utilizará para hacer la conexión a la fuente de alimentación será de cobre)

Un detalle importante, en cuanto a la colocación de la malla de acero, es que en ningún momento entre en contacto con la pieza a tratar. La separación del tanque la haremos mediante hojas de polietileno de alta densidad. Además, se coloca a los electrodos una protección de plástico compatible para evitar que no entren en contacto con el ánodo que rodea a la pieza.

\section{Electrolito}

Para conservar una pieza de hierro, ya sea forjado o fundido, terrestre o marino, es imprescindible evitar su contacto con tres elementos claves o factores que afectan a su deterioro, como son: humedad, oxígeno y cloruros. Por tanto, en la extracción de un objeto de hierro, como no es posible la eliminación total e inmediata de los cloruros, el ambiente ideal de conservación será un ambiente húmedo y libre de oxígeno. En la práctica esto se puede realizar sumergiendo las piezas arqueológicas en soluciones básicas del tipo hidróxido sódico a una concentración determinada del $2 \%$ en el caso del hierro, aunque también se pueden sumergir en sesquicarbonato sódico al 5\%. En estas soluciones básicas, en principio, conseguimos la estabilización del objeto, se evita cualquier posible proceso de corrosión, puesto que las piezas de hierro son estables a pH básico, el cual evita también cualquier acidez 
provocada por la presencia de los iones cloruros. Por otra parte, otra característica que presenta el hidróxido sódico es que al ser un electrolito fuerte, en solución se disocia completamente en sus iones y por tanto conduce más electricidad.

Para las piezas de bronce esta sustancia no es apta, ya que es perjudicial para este tipo de aleación metálica. Para ello utilizamos otro tipo de electrolito más adecuado y compatible con las piezas de bronce, como puede ser el sesquicarbonato de sodio. Este electrolito, que es una mezcla de carbonato de sodio y bicarbonato de sodio, se ha utilizado en una concentración del 5\%.

En todo caso, el electrolito tiene que ser una sustancia que genere un pH básico, el cual favorece la estabilización termodinámica de la pieza frente a la corrosión, según los diagramas de Pourbaix.

\section{Baño}

El baño a utilizar presentará una serie de características como son: material plástico no conductor, resistente a ácidos y álcalis.

\section{Fuente externa}

Debe ser regulable en intensidad y tiene que permitir que se puedan dar barridos de corriente suficientemente amplios.

Un término a definir que juega el papel más importante en la estabilización de los objetos metálicos mediante reducción electrolítica y eliminación de cloruros, es la densidad de corriente de aplicación. Se define densidad de corriente como la intensidad de corriente por unidad de área atravesada por dicha corriente:

\section{$i=I / A$}

La densidad de corriente utilizada en la reducción electrolítica se expresa como el número de amperios por unidad de área de superficie de los artefactos que se introducen en la célula electrolítica. La densidad de corriente que va desde 0,001 hasta 1 $\mathrm{amp} / \mathrm{cm}^{2}$ se ha propuesto para su uso en la limpieza electrolítica (PLENDERLEITH, 1956:195; PLENDERLEITH; TORRACA, 1968: 242; PLENDERLEITH; WERNER, 1971: 198; TOWNSEND, 1972: 252), pero guias para la aplicación específica de las densidades de corriente rara vez se dan.

Hamilton (1996) propone un rango de densidades de corriente de aplicación:

1-5 $\mathrm{mA} / \mathrm{cm}^{2}$ reducción de los compuestos ferrosos productos de la corrosión

$50 \mathrm{~mA} / \mathrm{cm}^{2}$ removimiento de cloruros

$100 \mathrm{~mA} / \mathrm{cm}^{2}$ limpieza mecánica

Otros autores, como Worth Carlin, experimentan con otros rangos de densidades que van desde los $50 \mu \mathrm{A} / \mathrm{cm}^{2}$ a los $200 \mu \mathrm{A} / \mathrm{cm}^{2}$.

Para la reducción electrolítica de las piezas hemos partido de una densidad de corriente de $50 \mu \mathrm{A} / \mathrm{cm}^{2}$. Esta densidad de aplicación

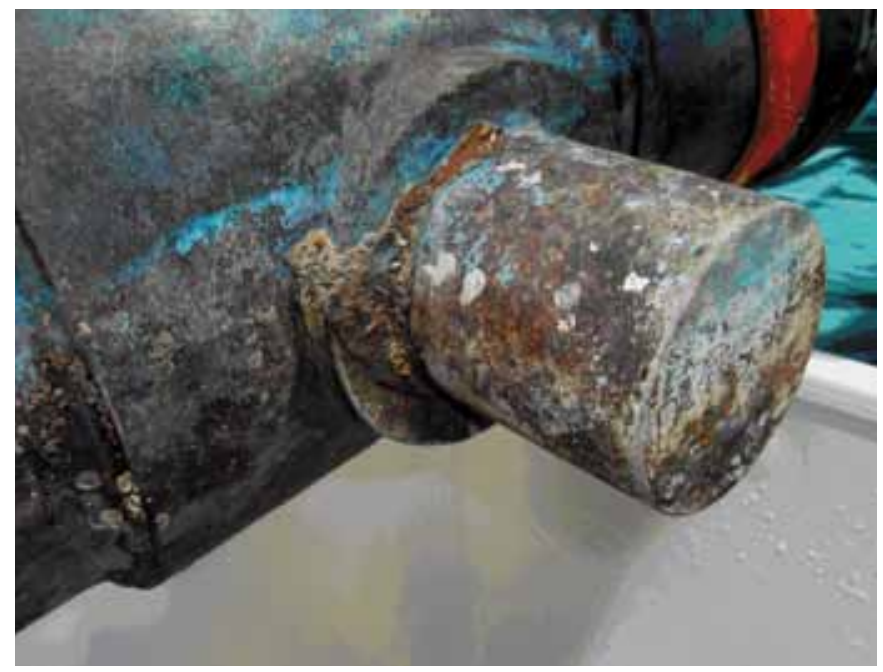

Detalle de los focos de cloruro. Fuente: CAS-IAPH

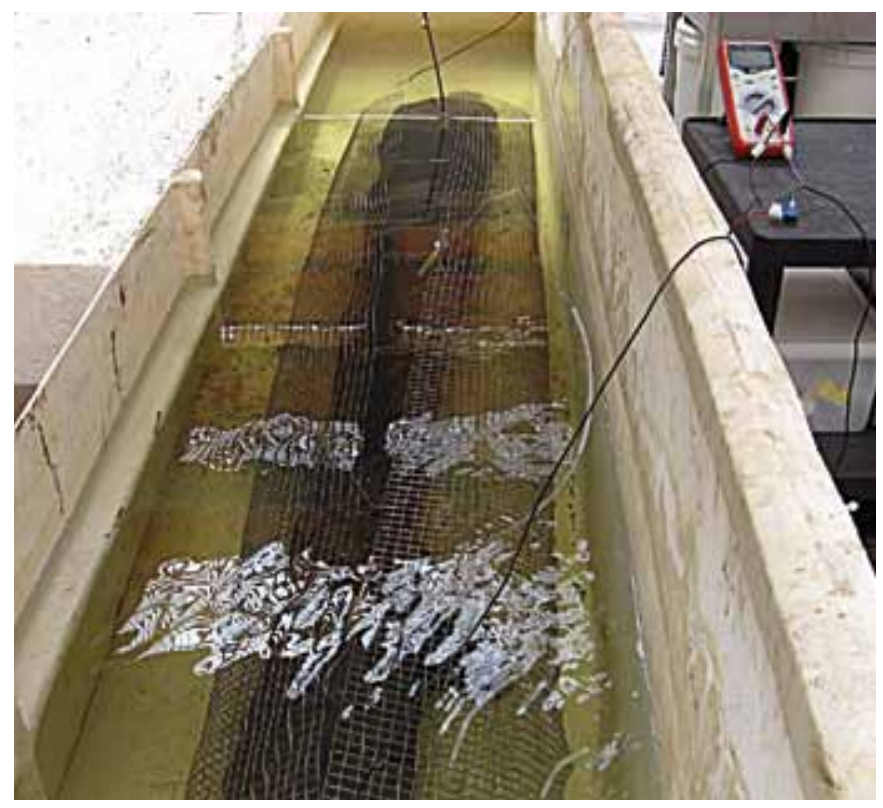

Celda electrolítica. Fuente: CAS-IAPH

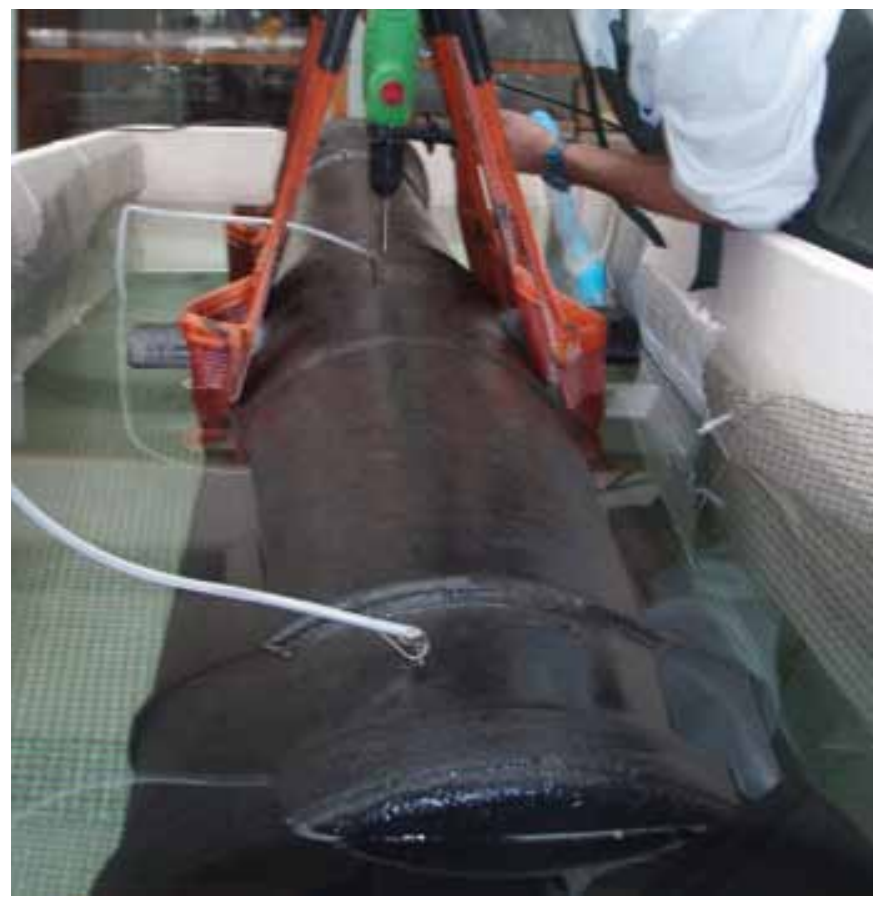

Colocación de electrodo en la parte posterior del cañón. Fuente: CAS-IAPH 

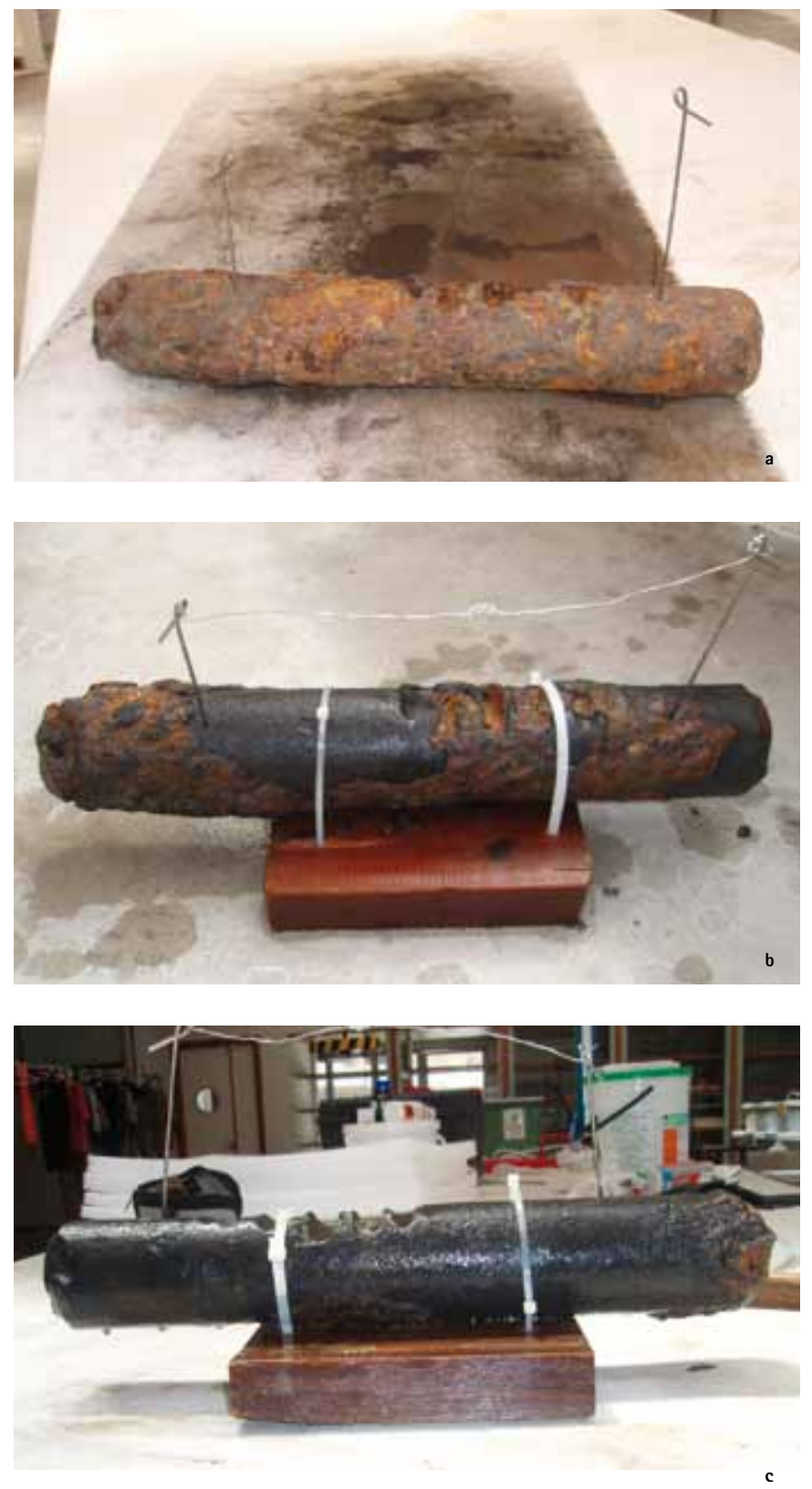

a, b y c. Limpieza electrolítica. Fuente: CAS-IAPH

$$
I(\mathrm{~mA})=J\left(\mathrm{~mA} / \mathrm{cm}^{2}\right) \times S\left(\mathrm{~cm}^{2}\right)
$$

$\mathrm{J}=50 \mu \mathrm{A} / \mathrm{cm}^{2}$

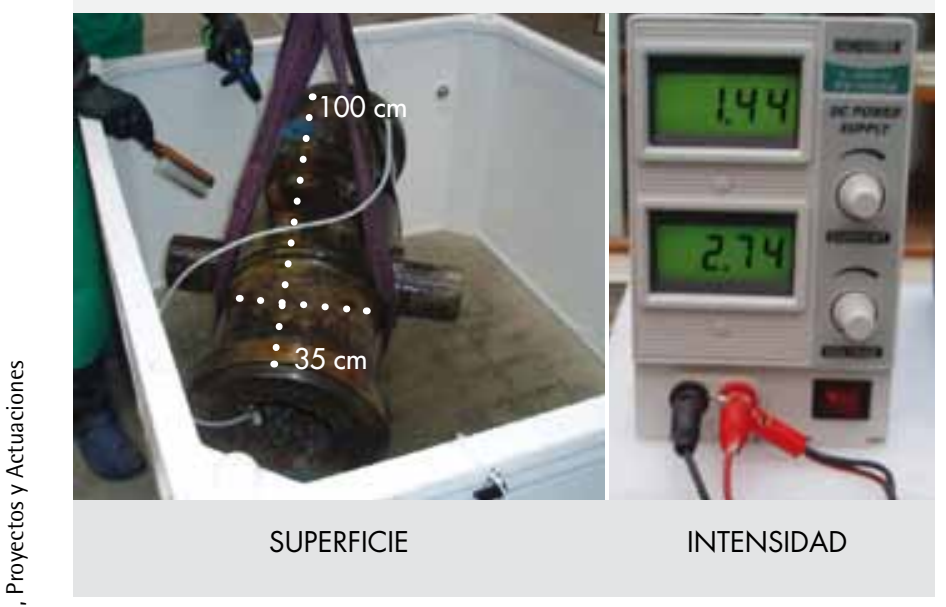

Densidad de corriente. Fuente: CAS-IAPH nos va a servir como un valor de seguridad con respecto al desprendimiento de hidrógeno, a partir de aquí hemos ido optimizando la intensidad de aplicación a cada pieza, hasta unos valores límites, cercanos a los que se produce la evolución de hidrógeno, con el fin, de que la reducción y la extracción de cloruros sea más rápida y eficaz.

La extracción de los cloruros y la estabilización de la pieza dependerán principalmente de la intensidad de corriente, el voltaje de aplicación y de la solución utilizada, los cuales, a su vez, dependen de otros parámetros como el tamaño y estado de deterioro del artefacto.

Hay autores que proponen que el área de superficie aparente para el cálculo de la intensidad de aplicación sea la sombra del área de uno de los lados del electrodo. Por tanto, las dimensiones del cátodo, es decir, la pieza metálica a la cual le aplicamos el tratamiento electrolítico con el fin de desconcrecionar, desalar y estabilizar, es otro factor importante a tener en cuenta a la hora de iniciar el tratamiento. En función del área del cátodo y de la densidad de corriente a aplicar, vendrá condicionada la intensidad de corriente eléctrica que se introduce en la celda electrolítica por medio de la fuente de alimentación externa. En este punto hay que tener en cuenta que por medio de la electrólisis se descompone la molécula de agua, migrando el oxígeno al ánodo y el hidrógeno al cátodo, desprendiéndose este último en forma de burbujas. Como uno de los problemas que se presenta en el tratamiento de las piezas metálicas es el desprendimiento de hidrógeno, con el fin de fragilizar lo menos posible a los materiales arqueológicos, se opta la mayoria de las ocasiones, por aplicar intensidades bajas de corrientes ya que se va a originar menos hidrógeno. En teoria, la reducción de metales no requiere ningún desprendimiento de hidrógeno, pero la evolución irregular de hidrógeno a partir de la superficie del metal es un indicador visual que fluye la corriente.

El desprendimiento vigoroso de hidrógeno indica una alta densidad de corriente que mecánicamente limpia el artefacto.

La superficie del cátodo la hemos calculado midiendo la pieza, su longitud y su anchura. No hace falta que las medidas de la superficie de la pieza sean tan exacta. Esto nos va a dar una idea sobre los valores de intensidad que debemos aplicarle inicialmente a la pieza. Como aplicamos densidades de corriente bajas, no vamos a tener problemas de desprendimiento de hidrógeno.

Como las intensidades y los voltajes que se han manejado son de valores muy bajos, se han diseñado unos dispositivos que funcionan como fuente externa, basados en la ley de Ohm, consistentes en:

- Cargadores de teléfonos móviles, transformadores y fuentes de alimentación de diversos aparatos. La mayoría tienen voltajes que van desde $5 \mathrm{~V}$ (cargadores de móviles) a 12V. 


\section{La sustitución de fuentes de alimentación profesionales por cargadores de móviles, fuentes de alimentación de ordenadores, transformadores de bajo voltaje, nos proporcionan un rango de intensidades más preciso para cada pieza metálica}

- Resistencias regulables de distintos ohmios, que van desde 100 ohmios hasta 100K.

- Amperímetros, que nos miden la intensidad de aplicación, y de donde parten las conexiones hacia el ánodo y el cátodo.

Dentro de la mejora de la eficacia de los métodos electroquímicos para la estabilización de piezas metálicas, con el fin de optimizar la técnica electrolítica, disminuir el tiempo de tratamiento y aumentar la velocidad y la concentración de iones cloruros difundibles, se propuso la colocación de calostatos en los baños electroquimicos, bombas de recirculación con potencias y caudales adecuados para cada pieza en tratamiento, desconcreción inicial para aumentar la porosidad, utilización de diferentes electrolitos y disolventes.

Durante el proceso debe monitorearse la concentración de cloruros $y$, de ser necesario, cambiar las soluciones hasta llegar a valores seguros. Estos valores algunos autores los colocan en 100 ppm, otros en 50 ppm. Nosotros hemos parado el proceso muy por debajo de estos valores, en torno a los 20 ppm. El realizar análisis de los iones cloruros presentes en la disolución estabilizadora es una herramienta útil e imprescindible para el control de la estabilización de las piezas metálicas. El seguimiento de decloruración se ha realizado mediante representaciones gráficas de las concentraciones de cloruros obtenidas en cada medición de la muestra tomada del baño electrolítico, frente al tiempo expresado en semanas.

El análisis indirecto que se realiza de la concentración de cloruros se podría acompañar de un estudio cualitativo de la reducción de los productos de corrosión por el tratamiento electrolítico aplicado y la consiguiente difusión de los cloruros, sería útil para saber el contenido total de cloruros en el interior de la pieza, pero esto solo es posible realizarlo tomando muestras en distintas zonas y profundidades de la pieza.

El análisis de los cloruros presentes en el baño electrolítico se ha llevado a cabo por métodos potenciométricos de medición directa mediante I.S.E., y por titulaciones argentométricas. El

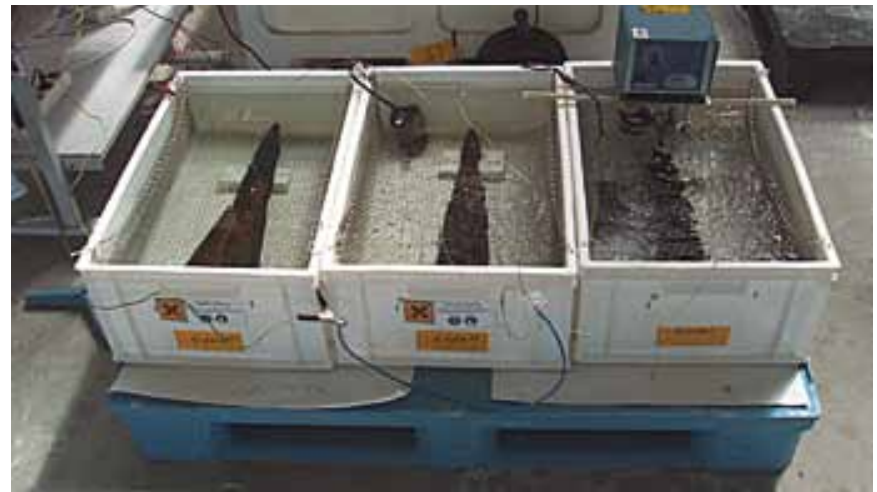

Mejora de la eficacia. Fuente: CAS-IAPH

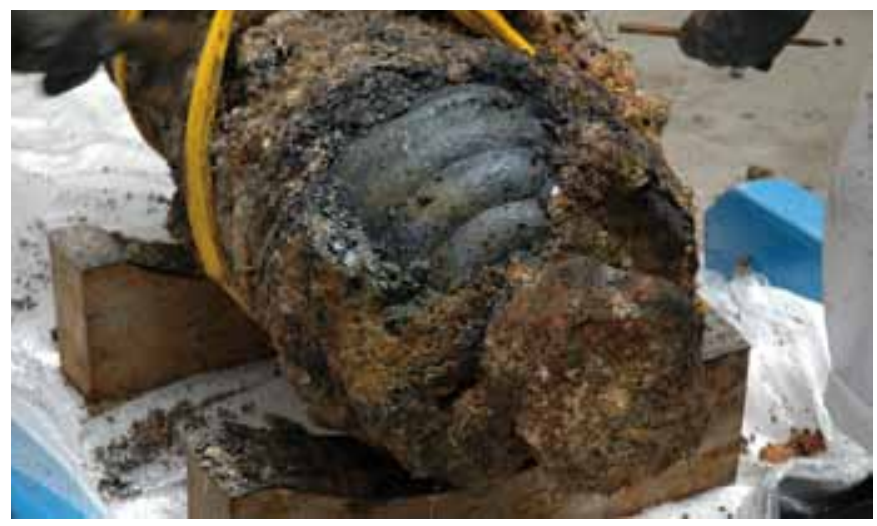

Desconcreción. Fuente: CAS-IAPH

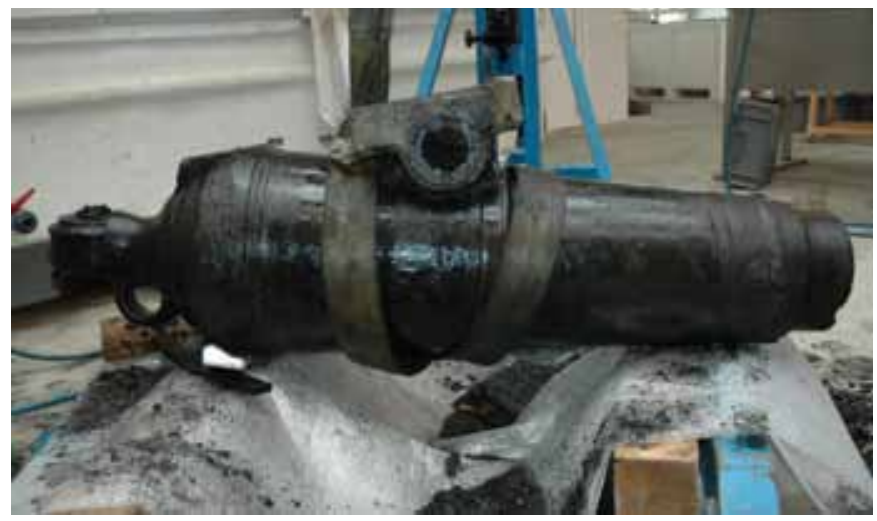

Consolidación. Fuente: CAS-IAPH

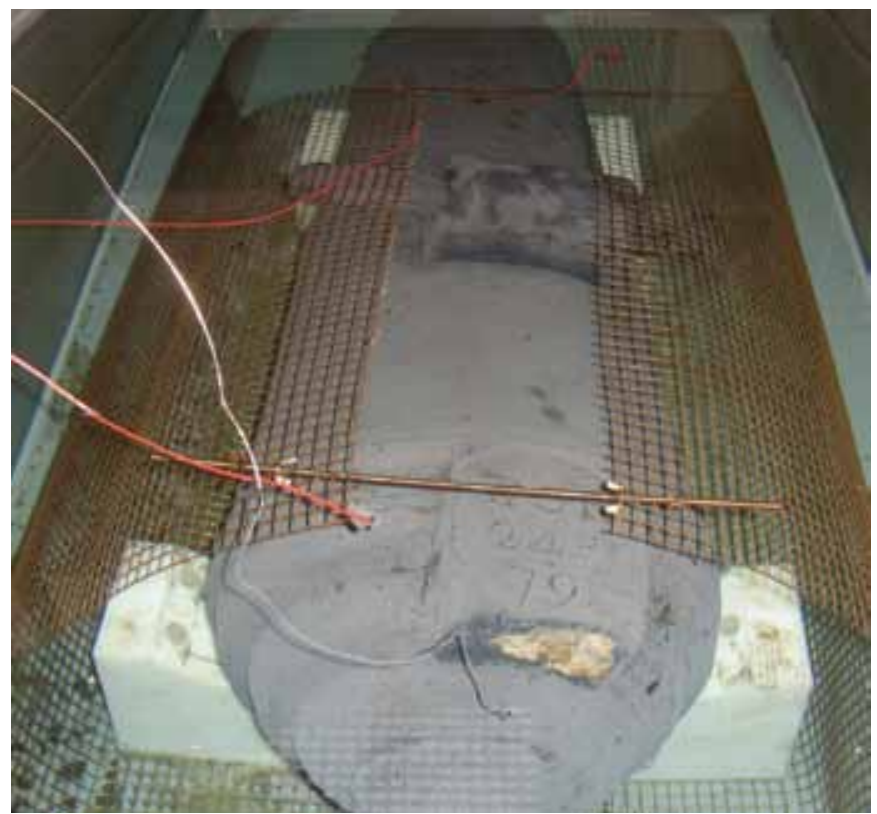

Tratamiento. Fuente: CAS-IAPH 


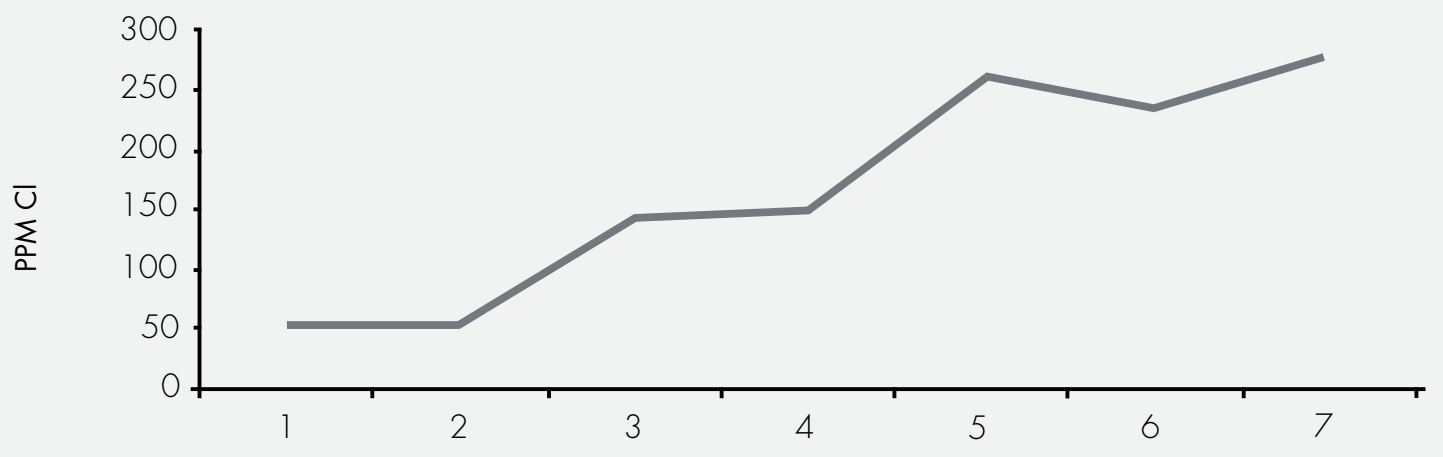

TIEMPO (SEMANAS)

Decloruración. Fuente: CAS-IAPH

método potenciométrico de análisis es la medida de un potencial con el fin de conocer la actividad o concentración de una sustancia en disolución. El objetivo de una medición potenciométrica es obtener información acerca de la composición de una disolución mediante el potencial que aparece entre dos electrodos. La medición del potencial se determina bajo condiciones reversibles, en forma termodinámica, y esto implica que se debe dejar pasar el tiempo suficiente para llegar al equilibrio, extrayendo la minima cantidad de intensidad para no influir sobre el equilibrio que se establece entre la membrana y la disolución muestra.

Los I.S.E. se utilizan en la potenciometría directa. Con esta técnica solo se necesita comparar el potencial del electrodo indicador cuando está inmerso en la solución del analito con el potencial que desarrolla el electrodo al estar en contacto con soluciones patrón de concentraciones conocidas del analito. Si la respuesta del electrodo es específica para el analito, como es frecuente, no es necesario hacer separaciones previas. También se pueden adaptar fácilmente a los análisis que requieran un seguimiento continuo y automático de los datos analíticos. Para las mediciones potenciométricas directas, el potencial de una celda se puede expresar en función de los potenciales que desarrollan el electrodo indicador, el de referencia y el potencial de unión liquida.

La respuesta del electrodo está relacionada con la actividad del analito más que con su concentración. Sin embargo, nos interesa conocer la concentración, y para determinarla con un método potenciométrico, necesitamos conocer el coeficiente de actividad.

El otro método de análisis ha sido la titulación argentométrica, que son titulaciones por precipitación que se realizan con nitrato de plata, producto que reacciona con los iones $\mathrm{Cl}^{-}$para dar lugar a la formación de un precipitado blanco de $\mathrm{AgCl}$. Una titulación potenciométrica consiste en medir el potencial de un electrodo indicador adecuado en función del volumen de titrante. La información que se obtiene con este método difiere de la resultante de la aplicación de medidas potenciométricas directas. Esta técnica presenta una serie de ventajas con respecto a las medidas potenciométricas directas. Como la medición del analito implica medir el volumen de titulante necesario para producir un cambio brusco en el potencial cercano al punto de equivalencia, este método no depende de medir los valores absolutos de $\mathrm{E}_{\text {celda }}$. En este aspecto, la titulación va a ser más fiable porque no se tiene la incertidumbre asociada al potencial de unión, ya que éste permanece casi constante durante la titulación. Los resultados de la titulación dependen más bien de contar con un titulante de concentración exactamente conocida. El potenciómetro simplemente señala el punto final; en este sentido, opera igual que un indicador químico. Los problemas debido a fallos del electrodo o que no dan una respuesta nerstiana son menos graves que los que presentan cuando el sistema de electrodo es el que mide el curso de una titulación. Asimismo, en una titulación potenciométrica tampoco es necesario saber exactamente el potencial del electrodo de referencia. Por otro lado, otra ventaja sería que el resultado del análisis se da en concentraciones del analito, aunque el electrodo responda a su actividad. Por lo tanto, la fuerza iónica no va a ser un factor importante a considerar en las mediciones.

El titrador utilizado en los análisis de cloruros es el modelo Crison SALT-Matic 23.

Para realizar una medición de la muestra, se introduce en ésta el electrodo combinado de plata conectado al titrador. Previamente se ha tenido que acidificar la muestra con ácido nitrico diluido con el fin de neutralizar. En segundo lugar, se añade nitrato de plata. Al ir añadiendo $\mathrm{AgNO}_{3}$ los iones $\mathrm{Ag}^{+}$se van uniendo en una relación 1:1 con los iones $\mathrm{Cl}^{-}$hasta completar las combinaciones posibles entre ellos en la disolución a valorar. La adición de $\mathrm{AgNO}_{3}$ se realiza de forma progresiva en pequeñas cantidades, llevándose 
a cabo mediciones en $\mathrm{mV}$ de la diferencia de potencial generada por el electrodo tras cada adición.

Dicho potencial varía en función de la reacción producida entre los iones $\mathrm{Ag}^{+}$añadidos y los $\mathrm{Cl}^{-}$presentes en la disolución en cada momento. El punto final se dará cuando se produzca un cambio brusco en el potencial. Los valores de cloruro obtenidos se pueden expresar de forma cuantitativa en ppm (partes por millón) o más correctamente, según el sistema internacional, en $\mathrm{mg} / \mathrm{l}$ (miligramos / litro).

El principal inconveniente que presenta un titrador frente a un pH-metro con electrodo selectivo de cloruros es el elevado precio.

Otros factores analizados, que están relacionados con la estabilización de los metales arqueológicos, son el pH del baño electrolítico y el potencial de corrosión tanto anódico como catódico, con el fin de contrastar por medio de los diagramas de Pourbaix, que el objeto metálico se encuentra en zona de pasivación o de inmunidad durante el tratamiento electroquímico.

\section{CONCLUSIONES}

Mediante la optimización de las técnicas electroliticas se ha conseguido una mejora de la estabilización del objeto. Se ha mejorado la eficacia debido, por un lado, a la reducción de costes mediante la sustitución de fuentes de alimentación profesionales que generan un alto rango de intensidades y voltajes innecesarios para el tratamiento llevado a cabo a bajas densidades de corriente, por un sistema constituido por cargadores de móviles, fuentes de alimentación de ordenadores, transformadores de bajo voltaje conectados a resistencias variables, que nos proporcionan un rango de intensidades más preciso para cada pieza metálica. Por otro lado, mediante el estudio de las leyes de difusión y la revisión bibliográfica, se han aplicado aquellos parámetros que afectan considerablemente de manera directa e indirecta, a la velocidad de difusión de los cloruros, con lo que se ha reducido el tiempo de tratamiento y se ha incrementado la decloruración de los objetos metálicos.

Se ha realizado un seguimiento exhaustivo de la estabilización de los objetos metálicos mediante la construcción de gráficas de desalación y las mediciones de $\mathrm{pH}$ y los chequeos de potencial tanto del cátodo como del ánodo.

Se ha mejorado el análisis de los cloruros mediante la utilización de un titrador, el cual, presenta una serie de ventajas frente a la utilización de electrodos selectivos de iones.

\section{Bibliografía}

BETHENCOURT, M.; GIL, A.; FERNÁNDEZ-LORENZO, C.; SANTOS, A. (2004)

Aplicación de tratamiento electroquímico a baja intensidad de corriente para la extracción de cloruros en objetos arqueológicos de hierro de procedencia subacuática. Observación de la evolución de fases mineralógicas mediante XRDRietveld. Revista de Metalurgia, vol. 40, n. ${ }^{\circ}$ 6, 2004, pp. 420-425

CARLIN, W.; KEITH, D.; RODRIGUEZ, J. (2001) Less is more: measure of chloride removal rate from wrought iron artifacts during electrolysis. Studies in Conservation, n. ${ }^{46}$, 2001, pp. 68-76

HAMILTON DONNY, L. (1996) Basic Methods of Conserving Underwater Archaeological Material Culture. Legacy Resource Management Program. Washington D.C.: U.S. Department of Defense, 1996

LAMY, C. (1997) Stabilisation d'Objects Archéologiques Chlorurés en Alliage Cuivreux. Définition des Conditions d'une Polarisation Cathodique à Potentiel Constant en Solution de Sesquicarbonate de Sodium 1\%. Rapport du Stage. Université de Nantes ISITEM, 1997

MACLEOD, I. D. (1987) Conservation of Corroded Copper Alloys: A Comparison of New and Traditional Methods for Removing Chloride lons. Studies in Conservation, n. ${ }^{3} 32$, pp. 25-40

NORTH, A.; PEARSON, C. (1978) Washing methods for chloride renoval from marine iron artifacts. Studies in conservation, n. 23,1978, pp. 174-186 PLENDERLEITH, J. (1956) The conservation of antiquities and works of art. London: Oxford University Press, 1956

PLENDERLEITH, J.; TORRACA, G. (1968) The Conservation of Metals in the Tropics. In The Conservation of Cultural Property. Museum and Monuments, n. ${ }^{11} 11968$, pp. $237-249$

PLENDERLEITH, J.; WERNER, A. (1971) The Conservation of Antiquities and Works of Art. Revised Edition. London: Oxford University Press, 1971 SKO0G, A. (2001) Quimica analítica. Séptima edición. Madrid: McGraw-Hill, 2001

TOWNSEND, P. (1972) Standard Conservation Procedures. In Underwater Archaeology; A Nascent Discipline. Museum and Monuments, n. 13,1972 , pp. 251-256 